\title{
Editorial
}

\section{GOVERNMENTS AND UNIVERSITIES}

\author{
BENJAMIN LEVIN* and NANCY SULLIVAN $\dagger$
}

... we must find new sources of funding for education. If we were to face a war we would certainly find the money. The challenge we now face in revitalizing and revamping our education system is, if less dramatic, even more important to the future of Canada than any of the wars we have fought. (Strong, 1987)

Provincial governments in Canada are estimated to have paid out some $\$ 7.7$ billion in grants to universities in 1987-88 (Statistics Canada, 1987), and this figure does not include all provincial payments for student aid, research, and special programs of various kinds or direct federal support of various forms. That this level of funding can be talked about as a crisis, or commonly referred to as "chronic underfunding", says something about the nature of governments and university relationships in Canada today. The two parties peer balefully at each other across a wide and apparently widening gulf.

The recent National Forum on Post-Secondary Education vividly illustrated the gap. Delegates from outside the post-secondary community talked of the many needs not currently being met by post-secondary institutions, and the need for those institutions to be more responsive than they are presently perceived as being. Delegates from post-secondary institutions on the other hand, who constituted a plurality (30\%) of the participants, talked repeatedly about the problem of lack of resources to meet these challenges.

For the universities, the issue seems obvious. University education is of fundamental importance to the country. It drives economic development and underpins social development. "The University of Manitoba is the Province's single most valuable human resource for economic growth", said a recent document produced by The University of Manitoba. "As the University's budget is ever-increasingly constrained, its role as the Province's major engine of economic growth will be weakened. The more the University is weakened, the more the economy will suffer" (University of Manitoba, 1987). Universities ought, it is argued, to have at least a steady share of such indicators as GNP or total government spending.

\footnotetext{
*Dr. Benjamin Levin is Executive Director of the Universities Grants Commision of Manitoba. $\dagger$ Nancy Sullivan is Assistant Deputy Minister, Post-Secondary, Adult and Continuing Education, Province of Manitoba.
}

Note: The opinions expressed in this paper are those of the authors in their private capacity, and do not necessarily represent the policy or opinion of the Government of Manitoba, the Department of Education, or the Universities Grants Commission. 
Moreover, providing access to universities for all who are qualified or wish to attend is seen as a fundamental tenet of a just society (if one dares to use that phrase in the 1980's), but providing places for the burgeoning population of adults wishing to enrol in universities, as well as the cohorts of sequential students, is an expensive business.

In making these arguments, universities have adopted with great skill the rhetoric of world competition. In the cutthroat international market, it is argued, which is so often coupled verbally with an emerging world of "hi tech", the development of knowledge will be more important than ever. The Strong quote above illustrates this argument at its most skilful.

The universities marry these arguments about their importance in the future with appeals to the past as well. They speak of the need to preserve and pass on the heritage of civilization. They emphasize the critical need for individual faculty members to be free to follow their interests, and they argue eloquently for the importance of providing each university not only with more dollars, but also with the maximum possible autonomy as to how to spend them.

From the standpoint of governments, the picture looks very different. Not only do some of the claims of universities appear somewhat exaggerated, but there are important additional considerations about which the universities are seen to be silent.

The first problem is, of course, the many pressures on the public purse, exacerbated quite often by the slow increase in government revenues from existing taxation sources. Sectors like education, with a large share of total spending, believe strongly that they need even more. Newer sectors with modest shares argue that their late emergence on the scene at a time of restraint should not deprive them of the resources needed to accomplish their important work. Naturally, there is no agreement among claimants as to whose needs will prevail. A colleague recently reported a conversation which occurred among the board of directors of a major hospital, arguing that education budgets should be cut to provide more money for health care! One wonders how many sectors have used Maurice Strong's analogy of war to support spending in their area - environmental groups? health care institutions? day care and child welfare? medical research? cultural organizations? municipal governments?

Governments are required, however reluctantly, to make allocation decisions among sectors. If they allocate less to post-secondary education than to health or social services, it is because that is their sense of priorities.

The post-secondary community has argued that these priorities are misplaced, that post-secondary education is fundamental to other development and so must be at the top of the list. This argument has not been totally persuasive to governments either in Canada, or by current data (Taylor, 1987), in other developed countries either.

There are several reasons for the lack of effect of the position advanced by post-secondary lobbies. The least important of these, in our view, is the way in which the case is put forward. Post-secondary representatives frequently say that 
they believe they would have more impact on governments if they "did a better job of telling their story" (Pierre, 1987). Our belief is that this is not true. The lobbies on behalf of post-secondary education are well organized and do communicate effectively. Moreover, because almost all politicians and senior officials have post-secondary education, a basic familiarity with the sector already exists. Such is not the case in other sectors such as energy, or natural resources, or environment, where most decision-makers will be, at first, quite ignorant of the field.

A more important concern to governments is the sense that expenditures in social services, such as education, can rise dramatically while at the same time, public satisfaction with the services declines in what Wildavsky (1979) called, speaking of health care, "doing better and feeling worse". To take an example, per pupil spending in Canada's public schools doubled in real terms, between the early 1970's and the early 1980's, while public support for and confidence in the schools declined. Health care expenditures rose dramatically, but there probably have never been so many complaints about access to health services. Post-secondary expenditures rose rapidly just before a period of high inflation, growing unemployment, and slow economic growth. These comments are made not to deny all link between spending and outcomes, but to point out the contingent nature with that link. At one time education was sold as the solution to social and economic problems. Post-secondary education still claims such positive impacts. Yet experience does not always support these claims. It is hard to think of an area of social service in which increased expenditure has, in fact, yielded all or most of the promised benefits. Is it, then, any surprise that governments do not altogether believe that still more dollars will lead to the promised land?

A further set of difficulties arises from the sense of many outside the post-secondary education community that post-secondary institutions are slow to change and unresponsive. The comment could, of course, be made about many, if not all, large institutions, but it carries a greater sting because post-secondary education advocates invoke dramatic societal change as grounds for the importance of post-secondary education, while seemingly, at the same time, resisting re-examination of their own practices and attitudes.

It might be worthwhile mentioning a few aspects of post-secondary activity which seem particularly troublesome in this regard.

- The great difficulties students often encounter in having their prior education recognized at a new institution. (This leaves aside entirely the question of credit for skills and knowledge gained outside formal education). This is a serious problem for Canadian students transferring, as well as for immigrants to Canada. Indeed, faculties or programs may even balk at recognizing courses offered elsewhere within the same province or even, on occasion, within the same institution.

- The insistence that quality is to be ensured by admission practices rather than by the outcomes of teaching and support services. Post-secondary institutions have not been willing, generally, to explore what could be done to allow those without 
the usual preparation to enter and succeed through improved pedagogy and better support services, despite evidence (Unruh, 1986) that much could be accomplished if the will were there. The insistence on admission standards suggested to one observer a parallel to hospitals insisting that they would admit only the healthy, since the sick required too much care, and might not get better in any case. - The slowness in adjusting to changes in the demographics of both actual and potential students. Even now not all programs are available part-time, while off-campus programs are still relatively infrequent. The specific needs of minorities, adults in the labour force, women re-entering the workforce, and other groups have been dealt with primarily through "special" programs, while the mainstream instructional and ongoing practices have hardly changed.

One could go on, but the point is not to denigrate post-secondary institutions. Rather, these are examples of the kinds of practices and attitudes which do more harm to the cause of post-secondary education than can ever be repaired by better public relations campaigns.

Nor are these comments meant to suggest that governments are on the side of the angels in their disputes with universities. Governments are, as all of us know, highly fallible. They operate under enormous pressure and usually with very limited time and information. Their nature requires that decisions be made, sometimes on highly complex and vitally important issues, in very short amounts of time. There is rarely an opportunity to give the issues the kind of careful and dispassionate analysis they may warrant. Governments are often caught trying to reconcile the irreconcilable, and to bring together contradictory points of view, or, at the least, to do things so as to offend as few people as possible (Levin, 1986). While some universities may sneer at this uncourageous approach to public policy, we do well to remember that it is a product of an open, democratic, and pluralist society. Just as we ask governments to understand certain aspects of the operations of universities as being "in the nature of things", so we must equally see the same truth in some of the less attractive features of governments.

These comments bring us squarely to the issue of the autonomy of universities. Canadian governments have been highly respectful of academic freedom for university faculty. The idea that faculty members must be free to study and teach according to their conscience is not in question.

There is, however, a considerable leap from this belief to the matter of the autonomy of universities as institutions very largely dependent on the public purse. As Harry Arthurs, President of York University, put it

Arguments about the legitimacy of government intervention are often no more than disagreements about the wisdom or faimess of government policy ... government intervention is inevitable once we accept the basic premise of government funding. (Arthurs, 1987, pp 12,14).

This does not mean that governments believe they should run universities, or dictate to them. In fact, governments in Canada have been very reluctant to impose 
directions on universities, partly because of their respect for tradition or autonomy, and partly from a belief that decision-making at the local level can be a very powerful benefit. The trend does appear to be in the direction of more government influence, primarily through targeted funding or regulation for particular purposes, although the vast bulk of support to Canadian universities continues to be in the form of unconditional block grants. An appeal to autonomy as a virtue in itself will not likely be effective, especially when autonomy may be seen as a cloak for a lack of willingness to reconsider policies and practices. Unless autonomy can be shown to produce valuable results (and we recognize the considerable ambiguity raised by that term), it will have less and less impact in the field of government/university relations. There are not likely to be major departures in university governance imposed by Canadian governments, but continued programmatic and financial pressure on universities seems a certainty.

All these tensions and difficulties do not mitigate a central fact: governments and universities are inextricably bound to each other. Universities have become central institutions for certain kinds of purposes, and these purposes are of great social importance. They are so important that they demand strong support, not only financially, but legally, and programmatically. (Often omitted in discussions of government/university relations is the importance of the legislative mandate given to universities to grant degrees and train professionals. See Skolnik, 1987).

The question to be asked, then, is how governments and universities can live and work together more productively; how the tensions which must inevitably exist between them can be as productive as possible. While there are no perfect solutions, some general direction can be suggested as a conclusion.

A first proposal would be for each party to rethink its stance or approach in relation to the other. Both general strategies and tactics need to be reconsidered in the light of current conditions, with a view not only to making one's case, but also to promoting a real understanding by each party of the reality of the other. One suspects that the attitudes of both sides, formulated for one set of circumstances or perhaps the result of accretion rather than any kind of reasoned formulation may not be the most suitable to today.

Secondly, since resources will continue to be limited, there will be increasing need to focus discussion on real issues, and to take every opportunity for successful collaboration rather than confrontation or attack. Of course, universities need to defend their essential values, and governments can be relied on to do the same. But mutual criticism, or even incessant complaint, cannot help either cause. Rather, greater use of negotiations, more joint venturing, and an effort to focus on what can be done instead of what cannot (Morrison, 1986) would be helpful.

The vehicles for this changed process are not presently in place. They will have to be invented, or developed based on those systems and organizations available. Such a change will require creativity, boldness, and goodwill. It will not be easy, but it is essential. 


\section{GOUVERNEMENTS ET UNIVERSITÉS}

\section{PAR BENJAMIN LEVIN* et NANCY SULLIVAN ${ }^{\dagger}$}

... nous devons trouver de nouvelles sources de financement pour l'enseignement. Si nous faisions face à une guerre, nous trouverions certainement l'argent; le défi qui se pose à nous aujourd'hui et qui consiste à revitaliser et à réorganiser notre système d'enseignement, est certes moins dramatique, mais encore plus important pour l'avenir du Canada que toutes les guerres auxquelles nous avons participé. (Strong, 1987).

On estime qu'au Canada les gouvernements provinciaux ont accordé quelque 7,7 millions de dollars de subventions aux universités en 1987-1988 (Statistique Canada, 1987), et ce chiffre ne comprend pas tout ce que les provinces payent d'aide aux étudiants, de recherche et de programmes spéciaux de toutes catégories, ni les diverses formes de financement accordées directement par le gouvernement fédéral. Que l'on puisse parler dans ces circonstances d'une situation de "crise", ou dire communément que l'on souffre "en permanence de financement insuffisant" montrent bien ce qui caractérise les relations qui existent actuellement entre les gouvernements et les universités. Les deux parties s'observent avec défiance et restent sur leurs positions de part et d'autre d'un gouffre profond qui semble se creuser sans cesse davantage.

Le Colloque national sur l'enseignement postsecondaire qui s'est tenu il y a peu de temps a montré ceci de façon très nette. Les délégués qui ne faisaient pas partie du secteur universitaire ont présenté les nombreuses exigences auxquelles les institutions postsecondaires étaient actuellement incapables de répondre et ont demandé à ces établissements d'en tenir compte davantage à l'avenir qu'à l'heure actuelle. D'autre part, les délégués des établissements postsecondaires qui représentaient la majorité relative des participants (30\%) ont dit à plusieurs reprises que c'était le manque de ressources financières qui les empêchait de répondre à ces exigences.

Du point de vue des universités, la question paraît évidente. L'enseignement universitaire est d'importance primordiale pour le pays. C'est un des leviers de l'expansion économique et la clef de voûte du progrès social. "L'Université du Manitoba est le seul outil, et par conséquent le plus précieux, que possède la province pour produire les ressources humaines capables de contribuer à la croissance économique", disait récemment un document publié par l'Université du Manitoba. "Plus le budget de l'université diminue, plus le rôle de cet outil

\footnotetext{
*Monsieur Benjamin Levin est directeur général de la Commission des subventions aux universités du Manitoba.

$\nmid$ Madame Nancy Sullivan est sous-ministre adjointe chargée de l'enseignement postsecondaire et de la formation permanente au ministère de l'Éducation de la province du Manitoba.
}

Remarque: Les idées exprimées dans cet article sont les idées personnelles des auteurs et ne représentent pas nécessairement le point de vue ou les objectifs du gouvernement du Manitoba, du ministère de l'Éducation ou de la Commission des subventions aux universités. 
essentiel au développement économique de la province s'affaiblit. Plus on affaiblit l'université, plus l'économie risque de souffrir." (Université du Manitoba, 1987). Les universités, nous dit-on, devraient au moins bénéficier d'un pourcentage stable soit du PNB du pays, soit du montant total des dépenses du gouvernement.

De plus, permettre à tous ceux qui en sont capables, ou qui le désirent, de suivre des cours dans des universités, est considéré comme un des principes fondamentaux d'une société juste (si toutefois l'on ose employer une expression de cette nature à notre époque); cependant, l'accueil de tous les adultes pleins d'espoir qui souhaitent s'inscrire à l'université, ainsi que du grand nombre d'étudiants en cours d'études est une entreprise qui coûte cher.

Les universités, lorsqu'elles ont recours à de tels arguments, montrent qu'elles ont adopté de façon très habile la rhétorique issue de la rivalité internationale. Dans le contexte de la concurrence acharnée que se livrent les divers pays sur le marché international, dit-on, et qui se double souvent de mots nés du monde nouveau des "experts de la technologie", l'approfondissement des connaissances deviendra plus important que: jamais. La citation de Strong présentée plus haut est une illustration des plus habiles de ce raisonnement.

En plus d'insister sur l'importance qu'elles auront à l'avenir les universités ont aussi recours au passé. Elles parlent de la nécessité que nous avons de préserver et de transmettre les traditions de notre civilisation. Elles insistent sur le besoin impérieux de laisser les professeurs d'université libres d'étudier ce qui les intéresse, et elles présentent avec éloquence tous les arguments qui font ressortir combien il est important non seulement de donner plus d'argent aux universités mais aussi de leur donner le maximum de liberté possible quant à la façon de dépenser cet argent.

Du point de vue des gouvernements, la situation semble bien différente. Non seulement les réclamations des universités paraissent sans garanties, mais il y a en outre d'autres aspects importants à considérer, aspects dont les universités ne parlent pas.

Le premier problème, bien sûr, vient de toutes les pressions que l'on fait sur ceux qui tiennent les cordons de la bourse des deniers publics, pressions d'autant plus fortes que les revenus du gouvernement provenant des taxes et impôts actuels augmentent lentement. Des secteurs tels que l'éducation, bien que bénéficiant déjà d'une grande part des dépenses gouvernementales, croient fermement qu'ils ont besoin d'encore plus d'argent. Les secteurs plus récents dont la part est plus modeste disent que le fait qu'ils soient nés plus tard et en période de restrictions budgétaires ne devrait pas les priver des ressources dont ils ont besoin pour accomplir un travail aussi important que le leur. Bien sûr, ceux qui adressent leurs réclamations au gouvernement ne discutent pas entre eux afin de savoir lesquels ont les besoins les plus pressants. Un de mes collègues me répètait récemment une conversation qui avait eu lieu au conseil d'administration d'un grand hôpital: on y disait qu'il faudrait couper les dépenses de l'éducation afin de donner plus d'argent aux organismes de santé! Je me demande par ailleurs combien de secteurs se sont servis de l'analogie avec la guerre que fait Maurice Strong pour faire valoir 
combien les dépenses de leur domaine sont essentielles - les organismes de protection de la nature ? les établissements de santé? les garderies et les services de protection des enfants? la recherche médicale? les organismes culturels? les municipalités?

C'est le rôle des gouvernements, quoi qu'il leur en coûte, de répartir les fonds entre les divers secteurs. S'ils accordent moins à l'enseignement supérieur qu'aux services sociaux ou aux services de santé, c'est que cela correspond à leur ordre de priorités.

La communauté universitaire s'est élevée contre ces choix qui sont, selon elle, mal faits; en effet, si l'enseignement postsecondaire est essentiel au progrès dans les autres domaines, il doit être le premier de la liste. Ce raisonnement n'a toutefois pas réussi à convaincre les gouvernements, que ce soit au Canada, ou dans les autres pays industrialisés, si l'on en juge par les données les plus récentes que nous ayons à ce sujet (Taylor, 1987).

Les raisons pour lesquelles le point de vue avancé par les groupes de pression des établissements postsecondaires n'a pas beaucoup d'effet sont diverses. La moins importante est, selon nous, la façon dont la situation nous est présentée. Les représentants des milieux universitaires disent souvent qu'ils jouiraient d'une plus grande influence sur les gouvernements s'ils "s'occupaient davantage de la manière dont ils expliquent la situation." (voir par exemple G. Pierre en 1987). Nous pensons que ceci est inexact. Les groupes de pression qui agissent au bénéfice de l'enseignement supérieur sont bien organisés et ils savent transmettre leur point de vue avec efficacité. De plus, presque tous les politiciens et les hauts fonctionnaires ont reçu une éducation supérieure, si bien qu'il y a dans ce milieu une bonne connaissance des universités, ce qui n'est pas toujours le cas dans les autres secteurs tels que l'énergie, les ressources naturelles ou l'environnement; bien souvent, ceux qui doivent prendre des décisions relatives à ces domaines n'en ont, pour commencer, qu'une faible connaissance.

$\mathrm{Ce}$ qui préoccupe davantage les gouvernements, c'est le sentiment que lorsqu'ils dépensent de l'argent pour renforcer les services sociaux tels que l'éducation, il arrive souvent qu'une augmentation des dépenses s'accompagne d'une baisse de l'appréciation du public vis-à-vis de ce service: c'est ce qu'a voulu dire Wildovsky en 1979 lorsqu'il a parlé, à propos des services de santé, des "améliorations grâce auxquelles la situation empire". Prenons un exemple: entre le début des années 70 et le début des années 80 , les dépenses nettes par élève des écoles publiques du Canada ont doublé, et dans le même temps, la confiance du public et son soutien vis-à-vis des écoles ont diminué. Les dépenses dans le domaine de la santé ont considérablement augmenté, et pourtant il n'y a sûrement jamais eu autant de plaintes qu'il y en a actuellement relativement à l'accès aux services de santé. Quand les dépenses pour l'enseignement postsecondaire ont rapidement augmenté, cette augmentation a été suivie d'une période de forte inflation, d'accroissement du chômage et de faible croissance économique. Ces exemples n'ont pas pour but de dire qu'il n'y a aucun lien entre ce qu'on sème et ce qu'on récolte, mais ils montrent bien combien ce lien est souvent fortuit. À une 
certaine époque, on soutenait que ce serait grâce à l'enseignement que l'on parviendrait à surmonter les problèmes économiques et sociaux. Les milieux universitaires soutiennent toujours ce point de vue. Pourtant, la réalité est tout autre. En fait on peut difficilement trouver un seul service public dans lequel l'augmentation des dépenses a effectivement produit les améliorations que l'on attendait d'elle. Faut-il donc s'étonner de constater que les gouvernements ne croient pas vraiment qu'une nouvelle augmentation de nos ressources nous permette d'atteindre notre but?

Mais il y a aussi d'autres difficultés, dues cette fois à l'idée que partage un grand nombre d'observateurs ne faisant pas partie du milieu universitaire; cette idée, c'est que les établissements postsecondaires n'évoluent pas facilement et sont lents à répondre à de nouvelles exigences. On pourrait bien sûr dire la même chose d'un grand nombre d'organismes de grande taille, et même peut-être de tous; toutefois, cette remarque a ici plus de mordant du fait que les porte-parole des universités invoquent les grands changements de société pour souligner combien l'enseignement postsecondaire est devenu indispensable, tout en se montrant réticents à revoir leurs méthodes et leurs attitudes.

Il serait peut-être bon de mentionner ici quelques aspects du fonctionnement des universités qui semblent particulièrement gênants de ce point de vue, il y a:

- les difficultés que rencontrent souvent les étudiants qui veulent faire reconnaître dans un nouvel établissement leurs études antérieures, (et l'on ne parle pas ici de la question des crédits que l'on pourrait accorder à un étudiant qui aurait acquis certaines connaissances en dehors d'un système éducatif reconnu). Ceci est un grave problème pour bon nombre d'étudiants canadiens qui changent d'université, ainsi que pour les immigrants qui s'installent au Canada. En fait, il arrive même que certaines facultés et certains programmes hésitent à reconnaître des cours offerts dans d'autres facultés ou programmes de leur propre établissement.

- l'insistance avec laquelle on veut maintenir la qualité des programmes à l'aide de procédures d'admission plutôt que par des techniques pédagogiques appropriées et des services de soutien. D’une façon générale, les établissements postsecondaires se sont montrés récalcitrants à étudier ce qu'ils pourraient faire pour permettre à ceux qui n'ont pas reçu la préparation prévue d'entrer à l'université et de réussir grâce à un encadrement pédagogique et à des services d'aide plus efficaces, malgré la preuve apportée par D. Unruh en 1986 que bien des choses pourraient être faites en ce sens si seulement on le voulait. Cette insistance pour conserver ces normes d'admission a fait dire à un observateur que c'était comme si les hôpitaux disaient qu'ils ne voulaient soigner que les bien-portants, les malades exigeant trop de soins et n'offrant pas de garantie que leur état va s'améliorer.

- la lenteur à s'ajuster aux changements démographiques tant par rapport aux étudiants inscrits qu'à ceux qui pourraient s'inscrire. Encore maintenant, on ne peut pas suivre n'importe quel programme à temps partiel, et les cours organisés ailleurs que sur le campus universitaire restent relativement rares. On répond aux besoins spécifiques des minorités, des adultes qui travaillent, des femmes qui 
retournent sur le marché du travail et d'autres groupes en créant des programmes "spéciaux", et l'on continue à enseigner les mêmes choses et à suivre les procédures habituelles, sans changement ou presque, dans la majorité des autres cours.

On pourrait continuer encore longtemps, mais notre but n'est pas de dénigrer l'enseignement supérieur. Ces exemples servent plutôt à montrer qu'il existe des attitudes et des habitudes qui font trop de mal à la cause de l'enseignement postsecondaire pour qu'elles puissent être compensées par de bonnes campagnes de relations publiques.

Ces commentaires ne visent pas non plus à suggérer que les gouvernements ont toujours raison. Nous savons tous combien les gouvernements peuvent se tromper. Ils agissent sous d'énormes pressions et souvent dans un minimum de temps et avec un minimum d'informations. Il est dans leur nature même d'avoir à prendre des décisions, quelquefois sur des questions vitales et très complexes, dans un espace de temps très court. Ils ont rarement la chance d'analyser les questions qui leur sont soumises avec le soin et l'impartialité qu'elles requièrent. Il faut souvent que les gouvernements tentent de concilier les contraires et de réunir des points de vue qui s'opposent, ou, du moins de choisir de faire ce qui offensera le moins de gens possible (B. Levin, 1986). Même si certaines universités pourraient être tentées de se moquer de cette façon plutôt lâche de prendre des décisions qui ont des répercussions sur le public, elles feraient peut-être bien de se souvenir que cette méthode est le produit d'une société fondée sur le pluralisme, l'ouverture d'esprit et la démocratie. De même que nous demandons aux gouvernements de comprendre que certaines façons de faire des universités sont "dans la nature des choses", nous devons accepter de reconnaître qu'il en est de même de certaines des caractéristiques gouvernementales les moins plaisantes.

Ces remarques nous amènent directement à la question de l'autonomie des universités. Les gouvernements ont toujours bien respecté la liberté intellectuelle des professeurs d'université. L'idée que ceux-ci doivent être libres d'étudier ce qu'ils souhaitent et d'enseigner ce que leur conscience leur dicte n'est pas remise en question.

Toutefois, de là à la question de l'autonomie des universités en tant qu'institutions, il y a un grand pas, surtout lorsqu'on considère que ces établissements dépendent largement des fonds publics. Comme l'a dit le président de l'université York, Harry Arthurs:

Les discussions qui consistent à demander si les gouvernements sont habilités à intervenir dans le fonctionnement des universités ne sont souvent rien d'autre que l'expression d'un désaccord relatif au bien-fondé ou à l'équité de certaines décisions gouvernementales...l'intervention du gouvernement est inévitable à partir du moment où on accepte qu'il finance les universités. (H. Arthurs, 1987 pp. 12-14)

Ceci ne veut pas dire que les gouvernements ont l'intention de diriger les universités et de leur dire quoi faire. En fait, au Canada, les gouvernements ont toujours hésité à imposer une certaine orientation aux universités, en partie parce qu'ils respectent leurs traditions et leur autonomie, mais aussi parce qu'ils croient que le fait de prendre des décisions à l'échelle locale peut être très bénéfique. 
Toutefois, il semble qu'à l'heure actuelle, les gouvernements exercent une influence accrue sur l'université, d'abord en finançant certains programmes de préférence à d'autres, mais aussi en élaborant certains règlements qui devraient permettre de répondre à des objectifs particuliers; malgré tout, la plus grande partie de l'aide aux universités canadiennes se fait encore par des subventions globales et non soumises à des conditions particulières. Un appel à l'autonomie fondé sur l'idée qu'elle représente, dans son essence même, une vertu, a peu de chances d'être efficace, surtout à un moment où l'autonomie peut être vue comme une façon de masquer le manque de bonne volonté du milieu universitaire face à une révision de ses règlements et de ses méthodes. À moins que l'autonomie ne montre qu'elle produit de bons résultats (et nous sommes bien conscients de l'ambiguité que peuvent créer ces termes), tout laisse croire qu'elle jouera un rôle de moins en moins grand dans les relations entre les gouvernements et les universités. S'il est peu probable que les gouvernements du Canada exigent le changement d'un grand nombre d'aspects de l'organisation des universités, il ne fait aucun doute qu'ils continueront à faire pression sur elles au moyen de programmes et de financement.

Toutes ces tensions et toutes ces difficultés ne rendent pas moins évidente une vérité essentielle: les gouvernements et les universités sont inexorablement liés les uns aux autres. Les universités sont devenues des organismes fondamentaux pour répondre à certains objectifs d'une grande importance sociale. En fait, ces objectifs sont si importants qu'ils exigent un soutien solide, non seulement financier mais aussi légal, au moyen de programmes gouvernementaux. (Parmi les questions souvent omises dans les discussions qui portent sur les relations entre les gouvernements et les universités, il faut signaler celle du rôle que la loi leur reconnaît, à savoir qu'elles sont habilitées à délivrer des diplômes et à former des spécialistes. Voir à ce propos M. Skolnik, 1987).

Alors, les questions qu'il faut poser sont les suivantes: comment les gouvernements et les universités peuvent-ils cohabiter, et comment doivent-ils s'y prendre pour travailler ensemble et obtenir de meilleurs résultats? Comment les tensions qui naîtront inévitablement de leurs relations pourront-elles être utilisées de façon à aboutir à des réalisations aussi positives que possible? S'il n'existe pas de solution miracle, nous aimerions, en guise de conclusion, proposer une orientation générale.

En premier lieu, nous aimerions que chaque partie réexamine ses positions, et sa façon de considérer l'autre partie. Les méthodes et les moyens employés doivent être repensés dans les deux cas à la lumière de la situation actuelle, non pas seulement avec l'intention de mieux faire valoir son point de vue, mais aussi afin qu'il en sorte une compréhension profonde pour chacune des parties de la réalité à laquelle l'autre doit faire face. On peut penser en effet que l'attitude des deux parties, fondée sur des idées toutes faites, ou peut-être sur une accumulation de principes plutôt que sur des arguments minutieusement repensés, n'est peut-être pas celle qui conviendrait le mieux aujourd'hui.

Deuxièmement, comme il faut s'attendre à ce que les ressources continuent à être limitées, il faudra bien orienter la discussion sur les vrais problèmes, et 
choisir, chaque fois que c'est possible, de collaborer efficacement plutôt que de s'opposer ou de s'attaquer les uns les autres. Sans doute les universités doivent-elles défendre leurs valeurs fondamentales, et l'on peut compter sur les gouvernements pour faire la même chose de leur côté. Toutefois, on ne saurait aller nulle part en se critiquant mutuellement et en se plaignant sans cesse. Il serait par contre bien plus utile de négocier davantage, de faire des tentatives conjointes et de concentrer ses efforts sur ce qui peut être fait plutôt que de se perdre dans ce qui est impossible (T. Morrison, 1986).

Les moyens grâce auxquels on pourrait changer la situation actuelle et établir ce type de relations n'existent pas encore. Il va falloir les inventer, ou les créer à partir des systèmes ou des types d'organisation actuels. Un tel changement exigera créativité, courage et bonne volonté. Ça ne sera pas facile, mais c'est absolument essentiel.

\section{REFERENCES}

Arthurs, H. (1987). The question of legitimacy. In C. Watson, Ed., Government and Higher Education - The Legitimacy of Intervention. Toronto: The Higher Education Group, OISE.

Duhamel, R. (1987, October). Remarks to Canadian university vice-presidents academic, Hamilton, Ontario.

Levin, B. (1986). Uneasy bedfellows: Politics and programs in the operation of government. Optimum, 17(1), 34-50.

Morrison, T. (1986, June), A certain ambiguity: University-government relations today. Paper for the CAUCE Conference, Ottawa, Ontario.

Pierre, G. (1987, November). Universities and the public agenda. University Affairs 28 (9).

Skolnik, M. (1987). State control of degree granting: The establishment of a public monopoly in Canada. In C. Watson, (Ed.), Government and Higher Education - The Legitimacy of Intervention. Toronto: The Higher Education Group, OISE.

Strong, M, (1987). Opening remarks to the National Forum on Post-Secondary Education. In Proceedings of the National Forum on Post-Secondary Education. Halifax: Institute for Research on Public Policy.

Statistics Canada. (1987). Advance Statistics of Education, 1987-88. Report 81-220. Ottawa: Minister of Supply and Services.

Taylor, W. (1987). The Role and Functions of the Universities. Paris: OECD Report 86(4).

University of Manitoba Bulletin. (1987, October 7). The University of Manitoba Multi-Year Financial Plan to 1992-93.

Unruh, D. (1987). Equality of access and equality of condition: Programming for success. In A. Artibise and W. Fraser, (Eds.), New Directions for Urban Universities: International Perspectives. Winnipeg: Institute of Urban Studies, University of Winnipeg, Report 18.

Wildavsky, A. (1979). Speaking Truth to Power: The art and craft of policy analysis. Boston: Little, Brown. 